\title{
Production, purification and characterization of hemolysins from Listeria ivanovii and Listeria monocytogenes Sv4b
}

\author{
Jürgen Kreft ${ }^{1}$, Dorothee Funke ${ }^{1}$, Albert Haas ${ }^{1}$, Friedrich Lottspeich ${ }^{2}$ and Werner Goebel ${ }^{1}$ \\ ' Institut für Genetik und Mikrobiologie, University of Würzburg, 8700 Würzburg, \\ and ${ }^{2}$ Max-Planck-Institut für Biochemie, 8033 Martinsried, F.R.G.
}

Received 13 June 1988

Revision received 30 August 1988

Accepted 30 September 1988

Key words: Hemolysin; Listeria

\section{SUMMARY}

In culture supernatants of both Listeria ivanovii and Listeria monocytogenes Sv4b, for the first time a hemolysin of molecular weight $58 \mathrm{kDa}$ was identified, which had all the characteristics of an SH-activated cytolysin, and which was therefore identified as listeriolysin O (LLO). In the case of L. ivanovii a second major supernatant protein of molecular weight $24 \mathrm{kDa}$ co-purified with LLO. However, the function of this protein has to be determined. In culture supernatants of $L$. ivanovii a sphingomyelinase and a lecithinase activity could be detected, both enzymatic activities together contributing to the pronounced hemolysis caused by $L$. ivanovii. The $\mathrm{N}$-terminal amino acid sequences of LLO and the $24 \mathrm{kDa}$ from $L$. ivanovii are shown.

Correspondence to: J. Kreft, Institut für Genetik und Mikrobiologie, University of Würzburg, 8700 Würzburg, F.R.G.

\section{INTRODUCTION}

The genus Listeria comprises a group of ubiquitous Gram-positive bacteria, some of which can cause severe infections (listeriosis) in animals and man [1], mostly in immunocompromised hosts. Pathogenic Listeria use professional phagocytic cells (monocytes and macrophages) of the infected host as the predominant site of multiplication. In normal cases infecting Listeria are eliminated upon activation of macrophages $[2,3]$, but this crucial step is impaired in the immunocompromised host.

All virulent Listeria (mostly L. monocytogenes) are hemolytic [4], whereas non-hemolytic Listeria are avirulent. This observation led rather early to the assumption that hemolysin production is an important virulence factor in Listeria infections.

It has recently been shown that $L$. monocytogenes Sv1/2a strain EGD produces a listeriolysin which belongs to the group of SH-activated cytolysins [6] (for a review see [5]), the prototype of which is streptolysin O (SLO) [7]. It could also be demonstrated that listeriolysin-negative transposon mutants of $L$. monocytogenes are virtually avirulent $[8,15]$ and that listeriolysin probably 
plays a key role in the escape of Listeria from the phagolysosomes of infected cells [9].

In the experimental mouse model system [10] the slightly hemolytic $L$. monocytogenes strains are quite virulent, whereas the extremely hemolytic $L$. ivanovii (formerly $L$. monocytogenes Sv5 or $L$. bulgarica) [11], is only moderately virulent.

\section{MATERIALS AND METHODS}

\subsection{Bacterial strains}

$L$. monocytogenes Sv4b (NCTC 10527), $L$. ivanovii (ATCC 19119, SLCC 23799), L. monocytogenes Sv1/2a (Mackaness, SLCC 5764), L. innocua Sv6a, and Rhodococcus equi (NCTC 1621) were obtained from the strain collection of the Institute of Hygiene and Microbiology, University of Würburg. L monocylogenes Sv1/2a (EGD) was from S.H.E. Kaufmann (Ulm) and Staphylococcus aureus was donated by $\mathrm{R}$. Lütticken (Cologne).

\subsection{Media and growth conditions}

Brain-heart infusion broth (BHI) (Gibco) or a defined Listeria medium (SLM) [12] were used and cultures were incubated at $37^{\circ} \mathrm{C}$.

\subsection{Purification of listeriolysin}

L. monocytogenes or L. ivanovii, respectively, were grown in BHI or SLM for $18 \mathrm{~h}$. The culture supernatants were cleared by centrifugation, concentrated about 50-fold by ultrafiltration (Amicon HPL10-20 or Millipore Pellicon PTGC) and subjected to thioldisulfide exchange affinity chromatography on thiopropyl-Sepharose 6B (Pharmacia) essentially as described by Geoffroy and Alouf [13]. In a further purification step gel filtration on Biogel P100 (Biorad) was used.

\subsection{Hemolysin assay}

Hemolytic activity of supernatants was determined in microtiter plates after serial two-fold dilution, and hemolytic activity of column fractions was determined in microliter tubes, using phosphate buffered saline (PBS), $\mathrm{pH} 6.0$ as a diluent. After $10 \mathrm{~min}$ preincubation with $10 \mathrm{mM}$ Dithioerythritol (DTE) sheep erythrocytes were added to a final concentration of $1 \%$. After $30 \mathrm{~min}$ at $37^{\circ} \mathrm{C}$ the release of hemoglobin was measured spectrophotometrically at $540 \mathrm{~nm}$. For the determination of the $\mathrm{pH}$ optimum, $5 \%(\mathrm{w} / \mathrm{v})$ sorbitol was added as stabilizer. In microtiter assays one hemolytic unit was defined as the reciprocal of the lowest dilution which gave complete hemolysis.

\subsection{Production of rabbit antiserum}

$0.25 \mathrm{mg}$ of purified $58 / 24 \mathrm{kDa}$ proteins in incomplete Freund's adjuvant were injected subcutaneously. The same amount of antigen was injected again 2, 4 and 5 weeks after the first immunization.

\subsection{SDS-polyacrylamide gel electrophoresis and im- munoblotting}

These were performed in $12.5 \%$ polyacrylamide slab gels, proteins were transferred to nitrocellulose filters by semi-dry blotting [14] and immunoreactive bands were visualized as previously described [15].

\subsection{Determination of N-terminal amino acid se- quence}

This was performed on a $470 \mathrm{~A}$ gas phase sequencer (Applied Biosystems). The phenylthiohydantoin derivatives were determined by HPLC as previously described [16].

\subsection{Sphingomyelinase activity}

This was measured by the method described by Gatt et al. [17] using trinitro-phenyl-aminolauryl(TNPAL) -sphingomyelin as a substrate. The concentration of the product was measured spectrophotometrically at $330 \mathrm{~nm}$. One unit was defined as hydrolysis of $1 \mu \mathrm{M}$ substrate $/ \mathrm{min}$ at $37^{\circ} \mathrm{C}, \mathrm{pH}$ 7.4. As positive control commercially available sphingomyelinase from $S$. aureus (Sigma) was used.

\subsection{Phospholipase $C$ activity}

This was determined by a modification of a previously described method [18] using $p$ nitrophenylphosphorylcholine as a substrate. PBS ( $\mathrm{pH} 7.0)$ was used in most experiments instead of $0.25 \mathrm{M}$ Tris- $\mathrm{Cl}(\mathrm{pH} \mathrm{7.2)}$ as no stabilization of Listeria phospholipase $\mathrm{C}$ by the latter buffer was 
noted. The enzymatic reaction results in the release of p-nitrophenol, which can be measured spectrophotometrically at $410 \mathrm{~nm}$. As positive control phospholipase $C$ from B. cereus (Sigma) was used.

\section{RESULTS}

Hemolysin production. In order to study the influence of growth media and growth phase on hemolysin synthesis, fresh BHI or the defined SLM-medium was inoculated with washed overnight cultures (BHI, $37^{\circ} \mathrm{C}$ ) of $L$. ivanovii or $L$. monocytogenes $\mathrm{Sv} 4 \mathrm{~b}$. The cultures were shaken at $37^{\circ} \mathrm{C}$, and samples were assayed at different times for cell density and hemolytic activity.

In both media hemolysin concentration peaked at $18 \mathrm{~h}$ and, in the case of $L$. ivanovii, the concentration of iron in SLM-medium had no significant effect on hemolysin concentration within the range of $1-10 \mu \mathrm{M}$ ferric iron (data not shown).

Purification of hemolysins. Bacteria were grown at $37^{\circ} \mathrm{C}$ for $18 \mathrm{~h}$ in $\mathrm{BHI}$. The purification protocol is outlined in Materials and Methods. Supernatant proteins with reactive $\mathrm{SH}$-groups were bound by thiol-disulfide exchange affinity chromatography to thiopropyl-Sepharose 6B [13]. Freshly regenerated Sepharose gave better results than the freeze-dried material obtained from the supplier, but in any case this step resulted in a significant loss of protein, as has also been noted by others [6]; a further purification step was performed by gel filtration on Biogel P-100 [13]. After each purification step the proteins contained in the hemolytic peak fractions were analyzed by SDS-PAGE. After the final step the hemolytic material of $L$. monocytogenes $\mathrm{Sv} 4 \mathrm{~b}$ consisted of a single, homogeneous protein of $M_{\mathrm{r}} 58 \mathrm{kDa}$ (Fig. 1, lane a). Hemolytic material from $L$ ivanovii contained two proteins of $M_{\mathrm{r}} 58 \mathrm{kDa}$ and $24 \mathrm{kDa}$ (Fig. 1, lane b). The $24 \mathrm{kDa}$ protein could only very incompletely be separated from the $58 \mathrm{kDa}$ protein by gel filtration on Biogel.

Identification of hemolysins. The hemolytic activity of fully reduced cytolysin preparations was severely inhibited by minor amounts of free cholesterol (final concentration much below the

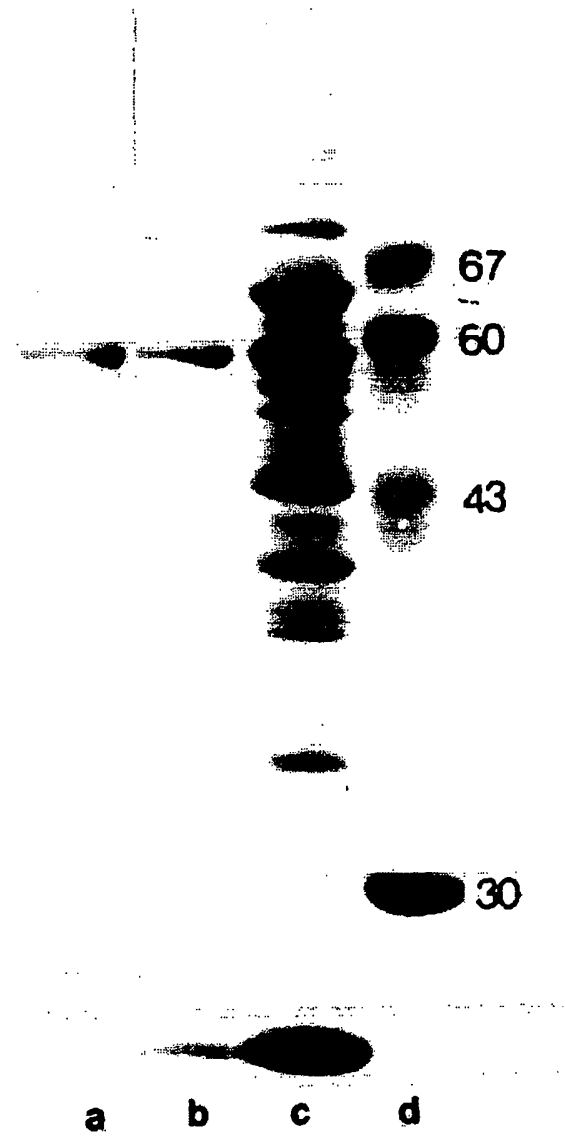

Fig. 1. SDS-polyacrylamide gel electrophoresis (12.5\% acrylamide, Coomassie blue staining). a, L. monocytogenes Sv4b, purified; b, $L$ ivanovii, purified; $c$, crude concentrated supernatant from $L_{\text {. }}$ ivanovii; d, molecular weight markers, figures on the right indicate $\mathrm{kDa}$.

limits of solubility). Inhibition by cholesterol was linear between $0-0.15 \mu \mathrm{M}$ and at $0.15 \mu \mathrm{M}$ inhibition was 90-95\%. Activation of the purified toxin preparations described above by DTE was linear in the range of $0-4 \mathrm{mM}$ and saturation was reached at $10 \mathrm{mM}$. Preincubation with $10 \mathrm{mM}$ DTE was used in all hemolysin assays. Stimulation by SH-reducing agents and inhibition by cholesterol is typical for SH-activated cytolysins of the SLO-type. In order to confirm this classification we performed immunoblots of these proteins from Listeria ivanovii and Listeria monocytogenes Sv4b using anti-SLO-serum. TCA-precipitated supernatant proteins from other Listeria strains and from $S$. pyogenes have been included in this ex- 


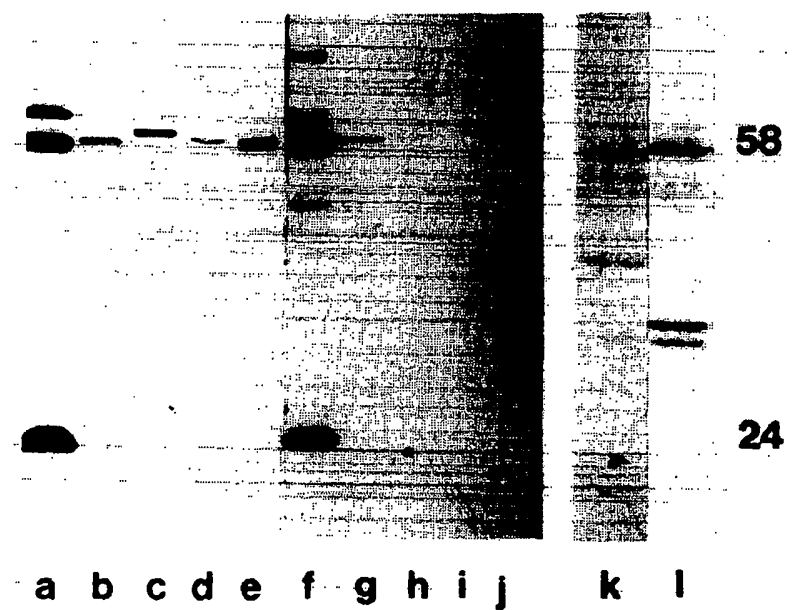

Fig. 2. Immunoblot of supernatant proteins after electrophoresis on $12.5 \%$ SDS-polyacrylamide gels. (I) Reaction with antiLLO-serum; (II) reaction with anti-SLO-serum. a, $f L$ ivanovii; b, g $L$ monocytogenes Sv $4 b$; c, h $L$ monocytogenes $S V 1 / 2 a$ (EGD); d, i $L$ innocua; e, $j L$ monocytogenes $1 / 2$ a (Mackaness); $k, 1 S$. pyogenes (SLO-producer). Figures on the right indicate the molecular weight in $\mathrm{kDa}$.

periment. Replicas of the immunoblots first probed with equine anti-SLO were subsequently tested with rabbit antiserum raised against the two-component hemolytic protein $(58 / 24 \mathrm{kDa})$ preparation from Listeria ivanovii. Both sera, anti-SLO and anti-Listeria ivanovii-hemolysin (anti-LLO), reacted in an almost identical fashion with the supernatant protein preparations from $L$. ivanovii (Fig. 2, lanes a, f). Also, in the culture supernatants from Listeria monocytogenes $\mathrm{Sv} 1 / 2 \mathrm{a}$
(Mackaness and EGD) and Sv4b cross-reacting proteins of $M_{\mathrm{r}} 58 \mathrm{kDa}$ clearly could be detected with both antisera (lanes $b-e, g-j$ ). Surprisingly the $24 \mathrm{kDa}$ protein from $L$. ivanovii not only reacted, as expected, with anti-LLO but also with anti-SLO. Both antisera reacted with streptolysin $O$ from $S$. pyogenes (Fig. 2, k-1). Finally, rabbit antiserum against the $24 / 58 \mathrm{kDa}$ proteins strongly inhibited hemolysis of sheep erythrocytes by concentrated culture supernatants from Listeria ivanovii in standard hemolysin assays (Hemolytic titers without anti-LLO or with $10 \mu \mathrm{l}$ pre-immune serum were $32768 \mathrm{U} / 50 \mu \mathrm{l}$, after addition of $10 \mu 1$ immune serum the titer dropped to $64 \mathrm{U} / 50 \mu \mathrm{l}$ ).

$N$-terminal amino acid sequences. We have determined the $\mathrm{N}$-terminal amino acid sequences of both the $58 \mathrm{kDa}$ protein (listeriolysin, 22 residues determined) and the $24 \mathrm{kDa}$ protein (19 residues determined) from $L$. ivanovii by microsequencing in a gas phase sequenator (Fig. 3).

Further biochemical and functional characterization. By isoelectric focussing the isoelectric points of the $58 \mathrm{kDa}$ protein (listeriolysin) and of the 24 $\mathrm{kDa}$ protein from $L$. ivanovii were determined as 7.2 and 4.9 , respectively. The pH-optima for the hemolytic activity of the purified cytolysins from $L$. monocytogenes $\mathrm{Sv} 4 \mathrm{~b}$ and $L$. ivanovii were determined as pH 5.0 and pH 5.5, respectively.

The classical way to distinguish between $L$. monocytogenes and $L$. ivanovii is their behaviour in the so-called 'CAMP'-test $[19,20]$ : Beta-toxin (sphingomyelinase) producing $S$. aureus or on the

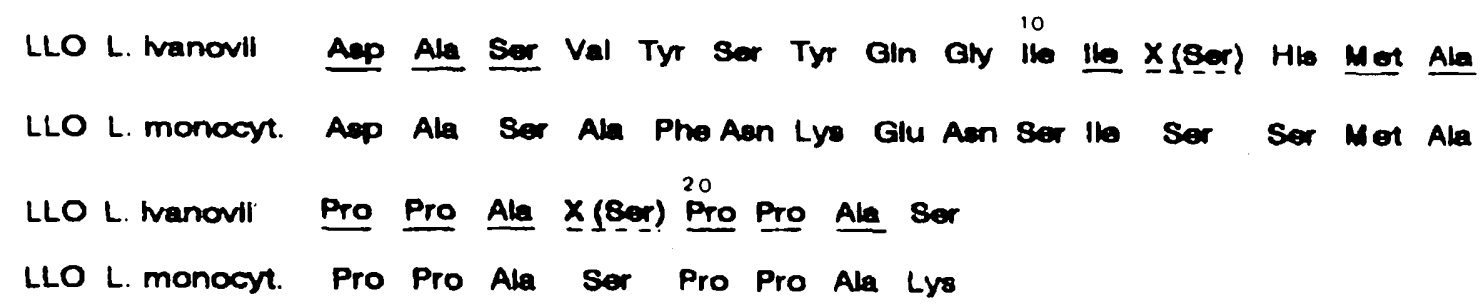

$24 \mathrm{KDa}$

\author{
Ala Aep lle Leu $x$ Pro Met Pro lle Aan Gin lle Phe Pro Alp \\ Prollew Alep Lou Ala
}

Fig. 3. $N$-terminal amino acid sequence of $58 \mathrm{kDa}$ protein (LLO) and $24 \mathrm{kDa}$ protein, both from $L$ ivanovii. An ' $\mathrm{X}$ ' means an amino acid which could not be identified (most presumably serine). Below the the amino acid sequence for LLO from $L$ ivanovii, the amino acid sequence for LLO from $L$. monocytogenes as deduced from the DNA sequence [21] is shown. 
other hand Rhodococcus equi cross-streaked with Listeria show zones of enhanced hemolysis at the intersection. L. monocytogenes (all serovars) reacts strongly with $S$. aureus and $L$. ivanovii reacts strongly with $R$. equi. Diffusion/reaction tests on blood agar plates using unfractionated, concentrated culture supernatants from (i) L. ivanovii, (ii) $S$. aureus and (iii) $R$. equi showed that in addition to the clear circular hemolytic zones surrounding the wells with supernatants from $L$. ivanovii or $S$. aureus (caused by alpha-toxin or listeriolysin, respectively), a lens-shaped hemolysis was visible in the region where diffusible products from $L$. ivanovii and $R$. equi or $S$. aureus overlapped and reacted with erythrocytes simultaneously or subsequently (Fig. 4). Enzymatic tests with 20-fold concentrated BHI culture supernatants from $L$ ivanoviti clearly showed a significant phospholipase $\mathrm{C}$ activity and a pronounced

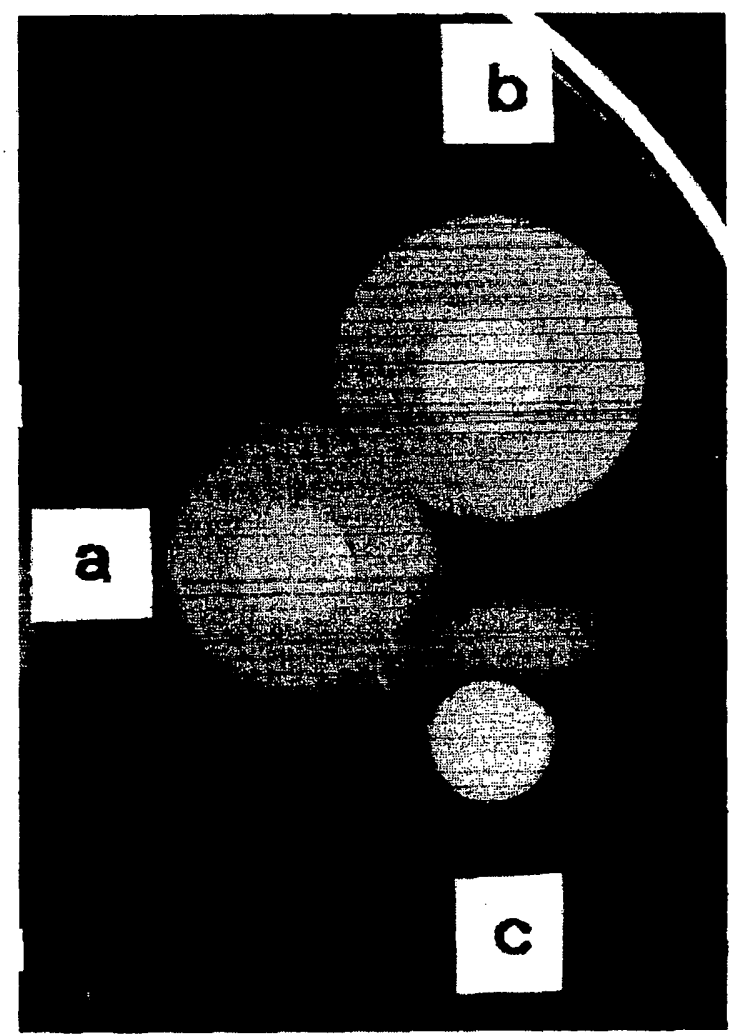

Fig. 4. Plate diffusion test on sheep blood agar plates. $50 \mu 1$ of 20 -fold concentrated culture supernatants $\left(18 \mathrm{~h}, 37^{\circ} \mathrm{C}\right.$ in $\left.\mathrm{BHI}\right)$ were applied per well. Incubation was overnight at 37 C. a, $L$ ivanovii; b, S. aureus; c, $R$. equi.
Table 1

\begin{tabular}{lcl}
\hline & $\begin{array}{l}\text { Sphingomyelinase } \mathrm{C} \\
\mathrm{U} / \mathrm{ml}\end{array}$ & $\begin{array}{l}\text { Lecithinase } \\
\Delta \mathrm{OD}_{410} / 16 \mathrm{~h}\end{array}$ \\
\hline Control (BHI) & 0 & 0 \\
L ivanovii & 360 & 0.81 \\
ATCC 19119 & &
\end{tabular}

10-fold concentrated supernatants of an $18 \mathrm{~h}$ culture were used

sphingomyelinase activity (Table 1). The enzymatic reaction to demonstrate phospholipase $\mathrm{C}$ activity in culture supernatants was slow, only after $16 \mathrm{~h}$ of incubation was a measurable activity detected. The value for the negative control remained zero even after this prolonged incubation. As a consequence of the long reaction time it did not seem reasonable to define the activity as units $/ \mathrm{ml}$, rather the difference in optical density is shown.

\section{DISCUSSION}

The results described above showed that relatively large amounts of a hemolytic protein with a molecular weight of $58 \mathrm{kDa}$ could be purified from culture supernatants of $L$ ivanovii and also L. monocytogenes Sv4b, grown in BHI for $18 \mathrm{~h}$ at $37^{\circ} \mathrm{C}$. These $58 \mathrm{kDa}$ proteins fulfilled all the requirements for $\mathrm{SH}$-activated cytolysins of the SLO-type and were therefore identified as listeriolysin O (LLO): (a) Hemolytic activity was stimulated by an SH-reducing agent (DTE), (b) hemolytic activity was inhibited by cholesterol added in micromolar amounts and, (c) they crossreacted immunologically with streptolysin $O$. Antiserum raised against $L$. ivanovii LLO cross-reacted with LLOs from other Listeria strains and with streptolysin $O$. Such a 'classical' listeriolysin $O$ has not been described for $L$. monocytogenes Sv4b so far. The pH-optimal for hemolytic activity of the toxins from both Listeria species were in the acidic range, which is in good agreement with the findings for LLO from $L$ monocytogenes Sv1/2a [6]. This further supports the assumption that LLO may play a key role in the escape of virulent Listeria from the acidic phagolysosomal environment. The $\mathrm{N}$-terminal amino acid se- 
quence of LLO from $L$. ivanovii was determined and shows good homology (at least 11 out of 22 amino acids, the first three amino acids are identical) to the deduced amino acid sequence of LLO from $L$. monocytogenes Sv1/2a [21].

The $24 \mathrm{kDa}$ exoprotein from $L$. ivanovii exhibited some unusual properties. It always copurified with LLO, but we could not determine if it bound to thiopropyl-Sepharose directly (via disulfide bonds) or via its association to LLO. In concentrated supernatants this protein apparently formed large complexes which could be dissociated only by SDS. So far we could not attribute any function to this protein. Plate diffusion tests also indicated that $L$ ivanovii secretes a factor which is phenotypically comparable to the CAMP-factor from Streptococcus agalactiae. Furthermore, both lecithinase and sphingomyelinase could be detected in $L$. ivanovii cultures supernatants. The lecithinase activity was rather weak, but the difference in activity between the culture supernatant sample and the blank was significant. This suggests that in L. ivanovii a bipartite hemolytic/cytolytic system is expressed, in addition to LLO. It has already been demonstrated that the combined action of sphingomyelinase and lecithinase can lyse mammalian cell membranes [22]. This second hemolytic system should contribute to the exceptionally high hemolytic activity and bizonal hemolysis, which is typically exhibited by $L$. ivanovii colonies on blood agar plates containing red blood cells with sphingomyelin-rich membranes, such as sheep erythrocytes.

\section{ACKNOWLEDGEMENT}

We are indebted to $\mathrm{H}$. Hof for several strains and the immunization and to J. Alouf for a generous gift of anti-SLO serum. We thank M. Dumbsky and S. Scherpf for their expert technical assistance and $\mathbf{M}$. Wuenscher for his help in preparing the manuscript. This work was supported by a grant from the Deutsche Forschungsgemeinschaft (SFB 105 and SFB 165).

\section{REFERENCES}

[1] Gray, M.L. and Killinger, A.H. (1966) Bacteriol. Rev. 30 , 309-382.

[2] Kaufmann, S.H.E. (1984) Infect. Immun. 45, 234-241.

[3] North, R.J. (1970) J. Exp. Med. 132, 521-529.

[4] Rocourt, J., Alonso, J.M. and Seeliger, H.P.R. (1983) Annu. Microbiol. (Inst. Pasteur) 134A, 359-364.

[5] Smyth, C.J. and Duncan, J.L. (1978) in Bacterial Toxins and Cell Membranes (Jeljaszewicz, J. and Wadström, T. eds.), pp. 129-183, Academic Press, New York.

[6] Geoffrey, C., Gaillard, J.-L., Alouf, J.E. and Berche, P. (1987) Infect. Immun. 55, 1641-1646.

[7] Alouf, J.E. (1980) Pharmac. Ther. 11, 661-717.

[8] Gaillard, J.-L., Berche, P. and Sansonetti, P. (1986) Infect. Immun. 52, 1843-1847.

[9] Gaillard, J.-L., Berche, P., Mounier, J., Richard, S. and Sansonetti, P. (1987) Infect. Immun. 55, 2822-2829.

[10] Hof, H. (1984) Med. Microbiol. Immunol. 173, 207-218.

[11] Seeliger, H.P.R., Schrettenbrunner, A., Pongratz, G. and Hof, H. (1982) Zbl. Bakt. Hyg., 1. Abt. Orig. A 252, 176-190.

[12] Siddiqi, R. and Khan, M.A. (1981) Zbl. Bakt. Hyg., I Abt. Orig. A 253, 225-235.

[13] Geoffroy, C. and Alouf, J.E. (1983) J. Biol. Chem. 258, 99689972.

[14] Khyse-Andersen, J. (1984) J. Biochem. Biophys. Meth. 10, 203-209.

[15] Kathariou, S., Metz, P., Hof, H. and Goebel, W. (1987) J. Bacteriol. 169, 1291-1297.

[16] Lottspeich, F. (1985) J. Chromatogr. 326, 321-327.

[17] Gatt, S., Dinur, T. and Bahrenholz, Y. (1978) Biochem. Biophys. Acta 530, 503-507.

[18] Kurioka, S. and Matsuka, M. (1976) Anal. Biochem. 75, 281-289.

[19] Christie, R., Atkins, N.E. and Munch-Petersen, E. (1944) Australian J. Exp. Biol. Med. Sci. 22, 197-200.

[20] Skalka, B., Smola, J. and Elischerova, K. (1982) Zbl. Bakt. Hyg., I. Abt. Orig. A 252, 17-25.

[21] Mengaud, J., Vicente, M.F., Chenevert, J., Pereira, J.M., Geoffroy, C., Gicquel-Sanzey, B., Baquero, F., Perez-Diaz, J.C. and Cossart, P. (1988) Infect. Immun. 56, 766-772.

[22] Zwaal, R.F.A., Roelofsen, B. and Colley, C.M. (1973) Biochem. Biophys. Acta 300, 159-182. 